\title{
A Novel Nanodrag Reducer for Low Permeability Reservoir Water Flooding: Long-Chain Alkylamines Modified Graphene Oxide
}

\author{
Hong Chen, ${ }^{1}$ Lin Xiao, ${ }^{1}$ Yuan $\mathrm{Xu}^{2}{ }^{2}$ Xiang Zeng, ${ }^{3}$ and Zhong-Bin Ye ${ }^{4}$ \\ ${ }^{1}$ State Key Laboratory of Oil and Gas Reservoir Geology and Exploitation, Southwest Petroleum University, Chengdu 610500, China \\ ${ }^{2}$ Research Institute of Natural Gas Technology, Petro China, Chengdu 610213, China \\ ${ }^{3}$ Sichuan Chuangang Group Corporation, Limited, Chengdu 610017, China \\ ${ }^{4}$ Sichuan Province Key Laboratory of Oil and Gas Reservoir of Applied Chemistry, Southwest Petroleum University, \\ Chengdu 610500, China
}

Correspondence should be addressed to Lin Xiao; 593412570@qq.com

Received 21 January 2016; Accepted 4 April 2016

Academic Editor: Victor M. Castaño

Copyright (c) 2016 Hong Chen et al. This is an open access article distributed under the Creative Commons Attribution License, which permits unrestricted use, distribution, and reproduction in any medium, provided the original work is properly cited.

Chemical modification of graphene oxide (GO) by grafting hydrophobic chains on the surface has drawn much attention nowadays in the academic world, and it was suggested that modified GO could lead to new functionalized materials with specific structure and different properties. In this paper, modified GO (M-GO) were synthesized by chemically grafting alkylamines with varying chain lengths on the graphene oxide surface. Successful grafting of alkylamines was confirmed using Fourier transform infrared (FTIR) spectra, X-ray diffraction (XRD), thermogravimetric analysis (TGA), scanning electron microscope (SEM), and Raman spectroscopy measurements. In addition, we investigated the properties of M-GO as nanodrag reducer in low permeability reservoir water flooding. Water contact angle (CA) measurements revealed that the hydrophobic nature of GO depended on the chain length of the grafted alkylamines. And flooding experiments showed that the hexadecylamine- and octadecylamine-modified GO had an ability to reduce water injection pressure and improve water-phase permeability of the low permeability reservoirs during water flooding. So the M-GO would have potential applications in oilfield exploitation.

\section{Introduction}

Graphene, an allotrope of carbon, has attracted extensive attention from both experimental and theoretical scientific communities due to its extraordinary properties, such as high structural strength, high surface-area to volume ratio, and excellent electrical properties [1-6]. Therefore, many exciting applications have been envisioned and demonstrated [711]. Graphene could be produced through several methods, such as mechanical exfoliation, chemical vapor deposition, electric arc discharge, and oxidation-dispersion-reduction. Among these methods, the oxidation-dispersion-reduction technique is widely used for it possesses great potential for larger-scale synthesis of graphene.

Various graphene-based materials could be conveniently synthesized from graphene oxide (GO) which is obtained from natural graphite flakes by the chemical oxidation [1215]. GO is a layered material featuring a variety of oxygencontaining functionalities with epoxy and hydroxyl groups on the basal plane and carbonyl and carboxyl groups along the edge [16]. On the one hand, these oxygen-containing functionalities could provide a platform for rich chemistry to occur both within the intersheet gallery and along sheet edge. On the other hand, it could render the graphene oxide layers hydrophilic and well dispersed in polar solvents to form colloidal suspensions $[17,18]$. Recently, more investigations have been focused on the surface property and wettability of graphene films $[19,20]$. Understanding the wettability of GO is important for the in-depth study of graphene-based functional materials. In general, the wettability of a solid surface is strongly influenced by both chemical composition and geometric structure (or surface roughness) [21-23]. 
To date, the reported organic functionalized methods of graphene include the reaction of GO with isocyanate groups, amide coupling between carboxylic acid and alkylamine, aryl diazonium functionalization, and the functionalization through the 1,3-dipolar cycloaddition of azomethine ylide [24]. Rafiee et al. reported that the surface roughness could be tuned by changing the solvent composition, which produced graphene films with the surface property ranging from superhydrophobic to superhydrophilic [25]. Zhang et al. indicated that the surface wettability of graphene film was greatly affected by the surface morphologies and achieved reversible transition from hydrophobicity to hydrophilicity via periodic alternation of ultraviolet irradiation and air storage [26]. Many significant studies have been done on the effect of amine modified GO on the surface wettability properties. Lin et al. prepared GO films with a contact angle of $53.8^{\circ}$, and it increased to $132.4^{\circ}$ after chemically grafting octadecylamine, which reduced the surface energy and enhanced surface roughness of the GO sheets [27].

The wettability of GO was transformed from hydrophilicity to hydrophobicity after surface modification. The pressure drop mechanism of hydrophobic nanometer materials is through hydrophobic nanometer adsorbing on the surface of porous walls and nanoparticles layer forming instead of hydrated layer. Consequently, wettability of pore wall turned from hydrophilicity to hydrophobicity, and slip effects on nanoparticles layer happened, and this decreased the flow resistance obviously and made fluid velocity obviously faster.

With the quick development of oil and gas exploitation, many middle and high permeability reservoirs have been in medium or high water cut period development stage, having a problem of lower water flooding recovery, and the low permeability reservoirs reserve account for a larger proportion. As a result, the low permeability reservoirs will be the major developing resources in the next development stage. Unfortunately, the development of low permeability reservoirs was limited by their poor properties, such as low permeability, low abundance, small porosity, low single well production, serious heterogeneity, and high residual oil saturation of water flooding, as well as the poor injectionproduction connectivity, which caused water injection pressure to increase rapidly and water intake capacity to decrease significantly during the water flooding process. In order to reduce water injection pressure and increase water injection volume, we should reduce the water flow resistance during water flooding.

In this paper, we wondrously found that the hydrophobic M-GO could be used as nanodrag reducer in the oilfield water flooding. We demonstrated a facile way for the synthesis of hydrophobic M-GO as nanodrag reducer through grafting octylamine (OA), dodecylamine (DDA), tetradecylamine (TDA), hexadecylamine (HDA), and octadecylamine (ODA) to GO surface. Also, we evaluated the performance of the $\mathrm{M}-\mathrm{GO}$ as drag reducer. With regard to the properties of drag reduction, the hexadecylamine- and octadecylaminemodified GO would find potential applications in low permeability reservoirs.

\section{Materials and Method}

2.1. Materials. Natural graphite powder (purity $\geq 99.85 \%$ ) was supplied by Shanghai Huayi Group Huayuan Chemical Co. Ltd., China. Potassium persulfate, phosphorus pentoxide, concentrated sulfuric acid, sodium nitrate, potassium permanganate, hydrogen peroxide, hydrochloric acid, THF, anhydrous ethanol, octylamine, dodecylamine, tetradecylamine, hexadecylamine, and octadecylamine were purchased from Chengdu Kelong Chemical Reagent Factory, China.

\subsection{Preparation of $G O$ and $M-G O$. GO was synthesized by} the improved Hummer's method [28].

M-GO, including OA-modified GO (GO-OA), DDAmodified GO (GO-DDA), TDA-modified GO (GO-TDA), HAD-modified GO (GO-HDA), and ODA-modified GO (GO-ODA), were conveniently prepared through the following steps. The as-prepared GO was dispersed in DI water $(1 \mathrm{mg} / \mathrm{mL})$ by ultrasonication for $2 \mathrm{~h}$ and centrifuging at $8000 \mathrm{rpm}$ for $30 \mathrm{~min}$ to remove aggregates. Ratio of reactants was GO $(1.0 \mathrm{~g})$ : alkylamine $(0.01 \mathrm{~mol})$. For example, $269 \mathrm{mg}$ of ODA was dissolved in $200 \mathrm{~mL}$ ethanol, and then the ODA solution was added in $100 \mathrm{~mL}$ GO dispersion at $60^{\circ} \mathrm{C}$ and stirred slowly for $12 \mathrm{~h}$. The mixture was filtered and washed with ethanol and DI water to remove unreacted alkylamine. Finally, the GO-ODA was dried at $60^{\circ} \mathrm{C}$ in a vacuum oven for 24 hours. Similar synthesis steps were followed in preparing GO-OA, GO-DDA, GO-TDA, and GO-HDA.

\subsection{Characterization. The FTIR characterizations of GO} and M-GO were performed using ThermoFisher Scientific Nicolet 6700 at ambient temperature. XRD measurements were performed directly on the powder sample using PANalytical XPERT-PRO diffractometer $(40 \mathrm{kV}, 40 \mathrm{~mA})$ with $\mathrm{Cu}(\lambda=1.54 \AA)$ irradiation. TGA was carried out with NETZSCH STA $449 F 3$. The heating rate was $10^{\circ} \mathrm{C} / \mathrm{min}$. Each time $\sim 5 \mathrm{mg}$ sample was measured in an aluminum crucible under inert gas atmosphere in $40 \sim 800^{\circ} \mathrm{C}$. Contact angle was determined using KRUSS DSA30. The slices of core were soaked into tetrahydrofuran solution which contain M-GO (20 mg/L). The solvent was heated and volatilized, depositing M-GO on the slices of core. Scanning electron microcopy (SEM) measurements were carried out on a field emission scanning electron microanalyzer (FEI Quanta 450) at an accelerating voltage of $20 \mathrm{kV}$. Raman spectroscopy was performed at ambient temperature using a HR800 Raman spectrometer at $532.2 \mathrm{~nm}$ wavelength incident laser light.

2.4. Evaluation of Core Flooding Experiment. All the displacement experiments were conducted on multifunctional chemical flooding physical simulation system (Haian Oil Scientific Research Apparatus Co., Ltd.). Schematic diagrams of experimental set-up were shown in Figure 1. The pres- 


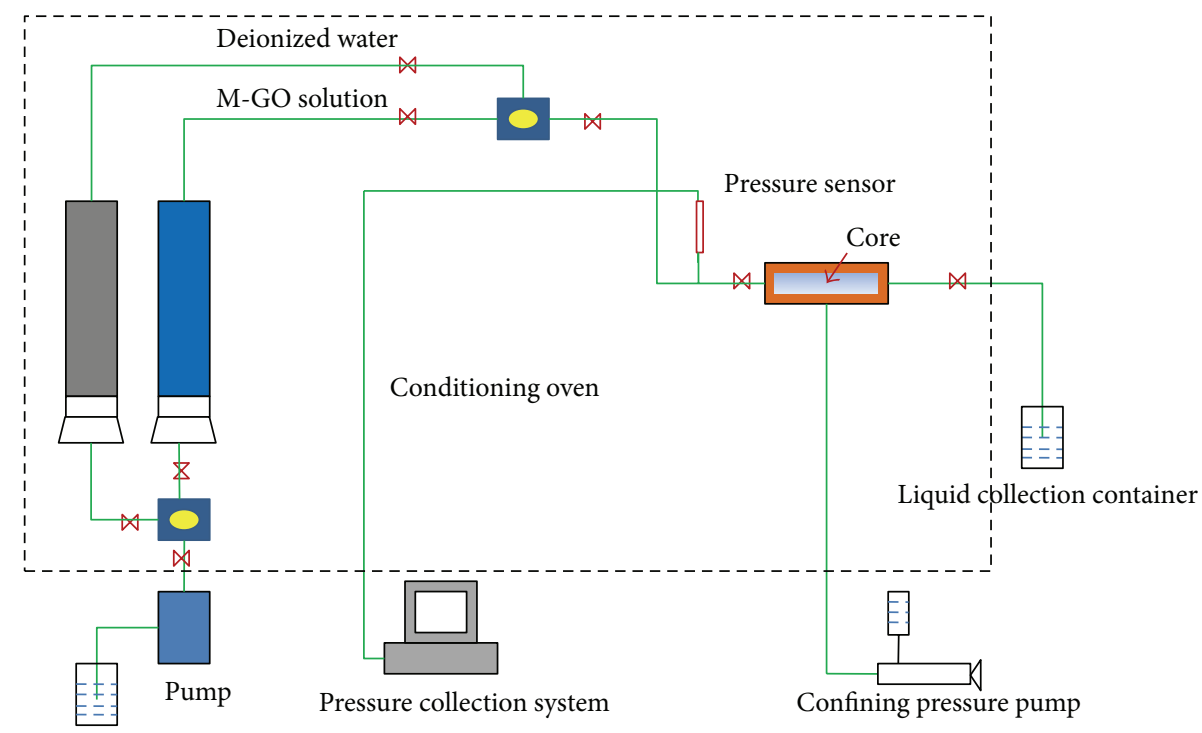

FIGURE 1: Schematic diagram of M-GO flooding experiment.

sure during the total injection process was recorded. The parameters of homemade cores were listed in Table 2. The fixed injection rate was $1 \mathrm{~mL} / \mathrm{min}$. The detailed process is as follows:

(1) Injection of DI water in the low permeability cores and determination of the initial water-phase permeability.

(2) Displacement of cores with 0 \# diesel fuel to set bound water.

(3) Displacement of cores with DI water again to set bound oil (enabling determination of the stable water flooding pressure $P_{1}$ ).

(4) Injection of 0 \# diesel fuel dispersion of M-GO.

(5) Injection of subsequent water flooding until there was no M-GO displaced out (enabling determination of the stable water flooding pressure $P_{2}$ ).

\section{Results and Discussion}

GO is a nonstoichiometric material prepared by improved Hummer's method, decorating with lots of oxygencontaining functional groups such as hydroxyl, carboxyl, carbonyl group and epoxy group. A previous study showed that hydrophobic long-chain alkyl groups in alkylamines and hydrophilic amine groups chemically reacted with the GO surface mainly via two types of reaction, namely, (1) amidation reaction between amine groups and carboxylic acid sites of GO and (2) nucleophilic substitution reactions between amine and epoxy groups on GO surface.

FTIR spectra of GO and M-GO were presented in Figure 2. For the GO spectrum, the major IR stretching vibrations at $3365 \mathrm{~cm}^{-1}, 1729 \mathrm{~cm}^{-1}, 1623 \mathrm{~cm}^{-1}, 1223 \mathrm{~cm}^{-1}$, $1047 \mathrm{~cm}^{-1}, 874 \mathrm{~cm}^{-1}$, and $826 \mathrm{~cm}^{-1}$ proved that GO contains

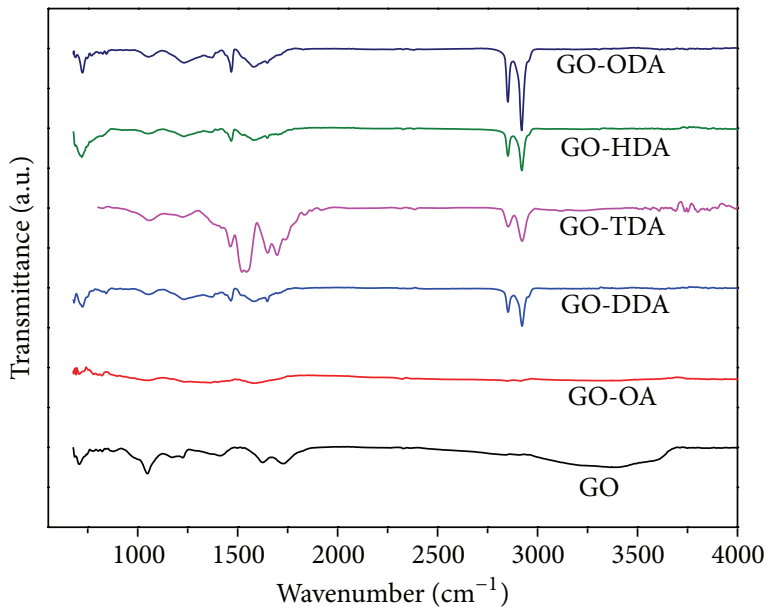

FIgURE 2: FTIR spectra of GO and M-GO.

hydroxyl, carboxyl, carbonyl, and epoxy groups. The existing of these four kinds of oxygen-containing functional groups indicated that GO was hydrophilic, so it could be well dispersed in water to form stable colloidal solution. After alkylamines modification, the peak at $3200 \mathrm{~cm}^{-1} \sim 3700 \mathrm{~cm}^{-1}$ which was attributed to water molecules weakened significantly; for long alkylamine chains (GO-TDA, GO-HAD, and GO-ODA), the peak disappeared completely. Meanwhile, the peak at $\sim 1729 \mathrm{~cm}^{-1}$ was related to stretching of the $\mathrm{C}=\mathrm{O}$ bond of carbonyl or carboxyl groups, which disappeared completely, and the peaks at $874 \mathrm{~cm}^{-1}$ and $826 \mathrm{~cm}^{-1}$ which were related to $\mathrm{C}-\mathrm{O}-\mathrm{C}$ bond intensity decreased. The changing indicated a strong reaction between $\mathrm{GO}$ and alkylamines. In addition, the amidation of carboxyl along the GO sheet edges with amino of alkyl amine dominated the reaction. The GO-DDA, GO-TDA, GO-HAD, and GO-ODA showed peaks 


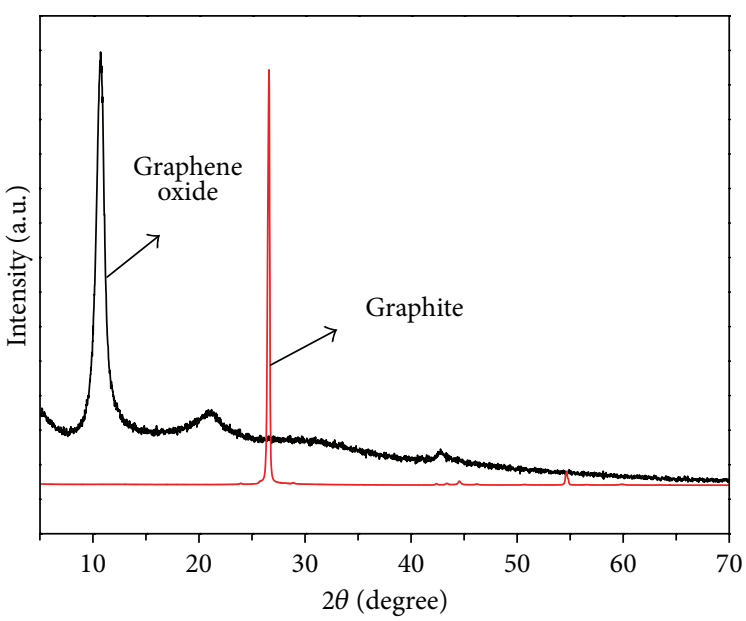

(a)

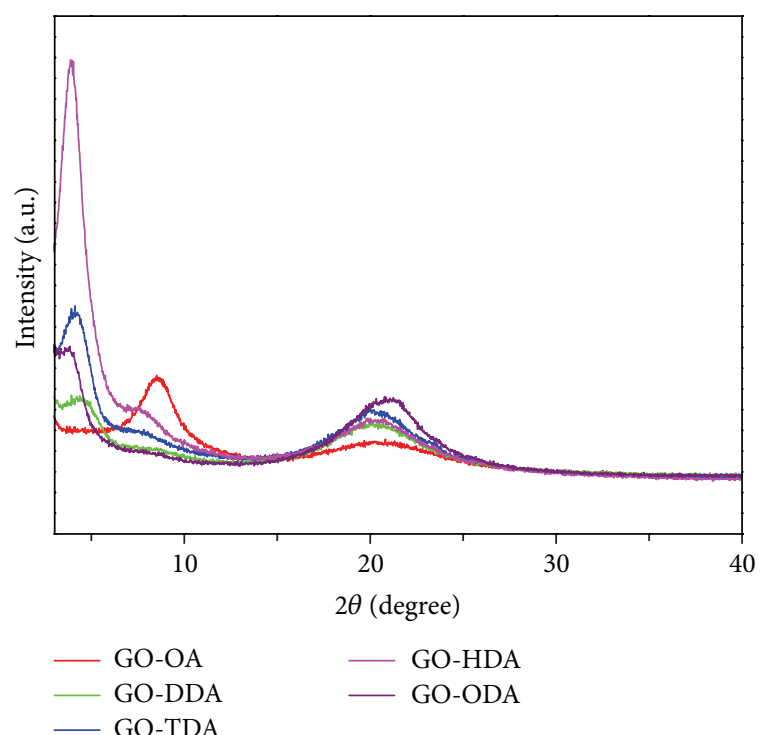

(b)

FIGURE 3: XRD patterns of GO, graphite (a) and M-GO (b).

at $1465 \mathrm{~cm}^{-1}$ and $1646 \mathrm{~cm}^{-1}$ attributed to $\mathrm{N}-\mathrm{H}$ and amide groups, respectively. The most significant change in FTIR spectrum of M-GO was the large intensity increase for peaks at $2920 \mathrm{~cm}^{-1}$ and $2850 \mathrm{~cm}^{-1}$, corresponding to the stretching of $\mathrm{C}-\mathrm{H}$ bond, which indicated the successful introduction of long hydrocarbon chains into GO sheets.

The XRD pattern of M-GO was shown in Figure 3. For the GO, the (001) peak appeared at $2 \theta=10.6^{\circ}$ (Figure 3(a)), indicating an interlayer spacing of $0.83 \mathrm{~nm}$. After modification, the GO (001) diffraction peak shifted forward (Figure 3(b)); for instance, GO-OA, GO-DDA, GO-TDA, GO-HAD, and GO-ODA showed a broad peak at $2 \theta=8.5^{\circ}, 4.6^{\circ}, 4.1^{\circ}$, $3.9^{\circ}$, and $3.6^{\circ}$, respectively. According to Bragg equation, the corresponding distance between two adjacent M-GO sheets was $1.04 \mathrm{~nm}, 1.92 \mathrm{~nm}, 2.15 \mathrm{~nm}, 2.26 \mathrm{~nm}$, and $2.42 \mathrm{~nm}$, which were all larger than that of original GO $0.83 \mathrm{~nm}$ due to the successful grafting long hydrocarbon chain onto GO sheet. At the same time, it could be found that the XRD pattern of M-GO showed a new peak around $20 \sim 21^{\circ}$ closing to pristine graphite (002) diffraction peak (around $26^{\circ}$, Figure 3(a)), which resulted from the disordered stacking of some reduced oxide-containing groups of GO sheets.

Figure 4 showed the TGA curves of GO and GO-ODA. Below $100^{\circ} \mathrm{C}$, GO exhibited a $\sim 11 \%$ weight loss, which could be attributed to the evaporation of water trapped between hydrophilic GO sheets, while the M-GO showed a significant decrease of weight loss, GO-OA only lost 5.5\%, and the other four kinds of $\mathrm{M}-\mathrm{GO}$ kept at a lower level around $2 \% \sim 3 \%$, indicating an enhanced hydrophobicity that minimized the amount of adsorbed water compared to pristine GO. Increasing the chain length of the grafted amines resulted in the decrease of weight loss below $100^{\circ} \mathrm{C}$. In addition, the GO showed nearly $39 \%$ weight loss from $100^{\circ} \mathrm{C} 300^{\circ} \mathrm{C}$; however, the M-GO only lost $20.1 \%, 21.8 \%, 19.35,17.2 \%$, and

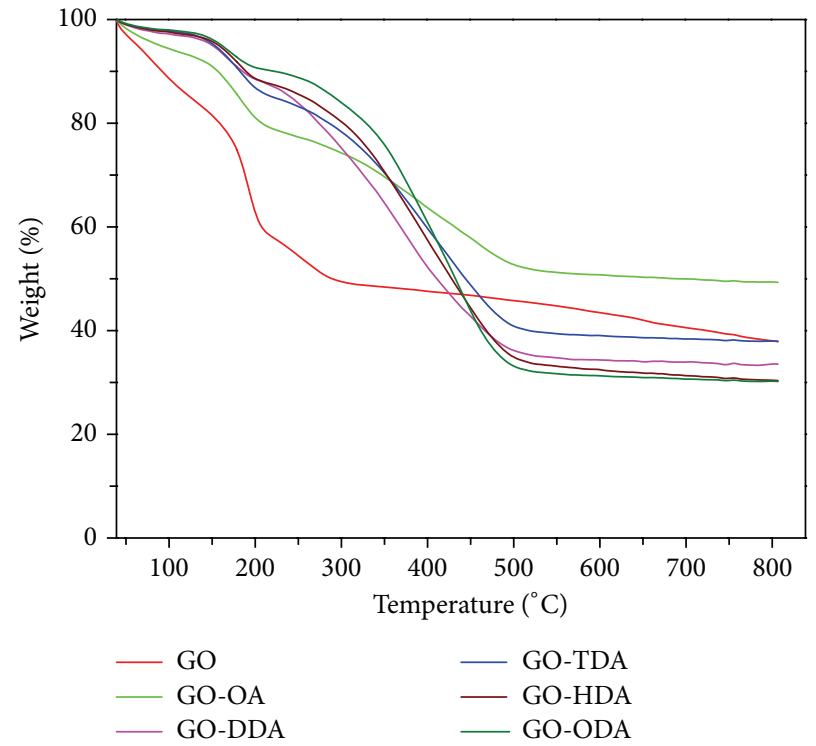

Figure 4: TGA curves of GO and M-GO.

$13.9 \%$, which was assigned to the decomposition of some oxide-containing groups. So accompanying modification procedure, a part of oxide-containing groups was reduced, which was in accord with the result of XRD. The thermal stability of M-GO increased with chain length of alkylamines below $400^{\circ} \mathrm{C}(\mathrm{GO}-\mathrm{OA}<\mathrm{GO}-\mathrm{DDA}<\mathrm{GO}-\mathrm{TDA}<\mathrm{GO}-$ $\mathrm{HDA}<\mathrm{GO}-\mathrm{ODA})$. However, when the thermal decomposition temperature exceeded $450^{\circ} \mathrm{C}$, the thermal stability of MGO became opposite to that of below $400^{\circ} \mathrm{C}$. M-GO showed a higher weight loss rate than GO except GO-OA, because the $\mathrm{C}-\mathrm{N}$ bond began to thermally decompose, and the long 


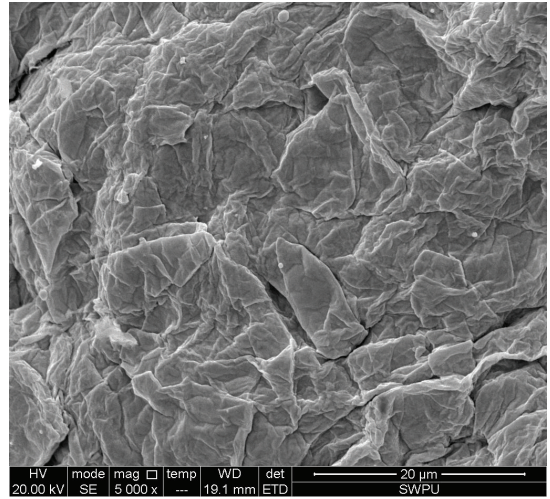

(a)

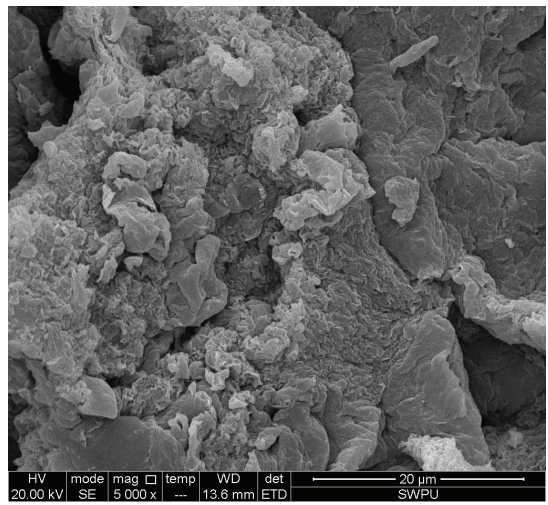

(d)

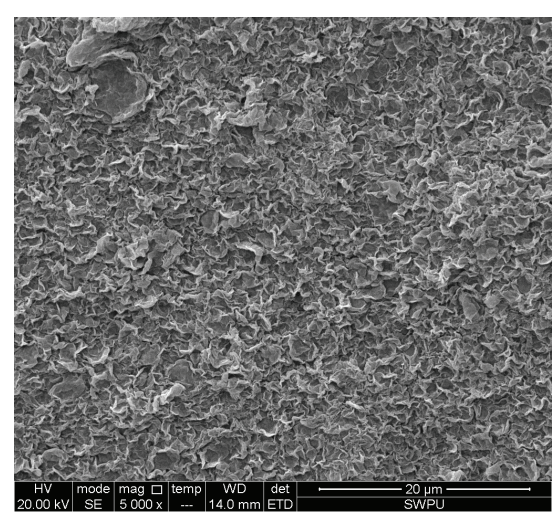

(b)

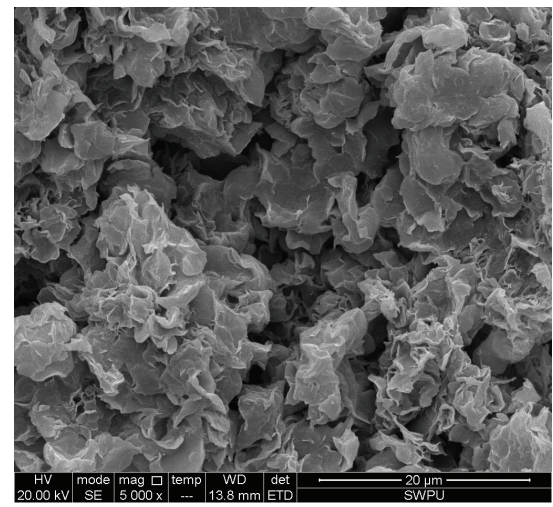

(e)

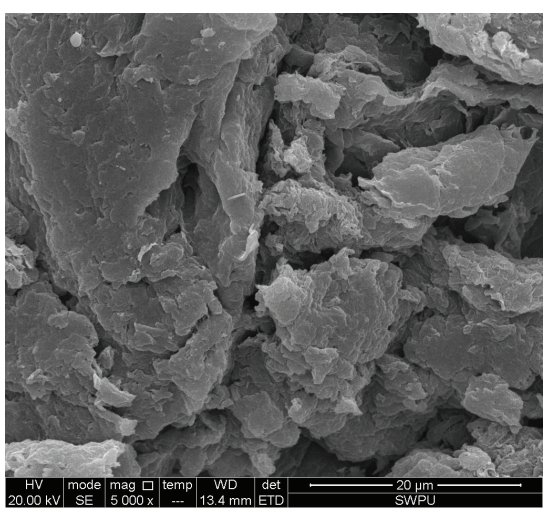

(c)

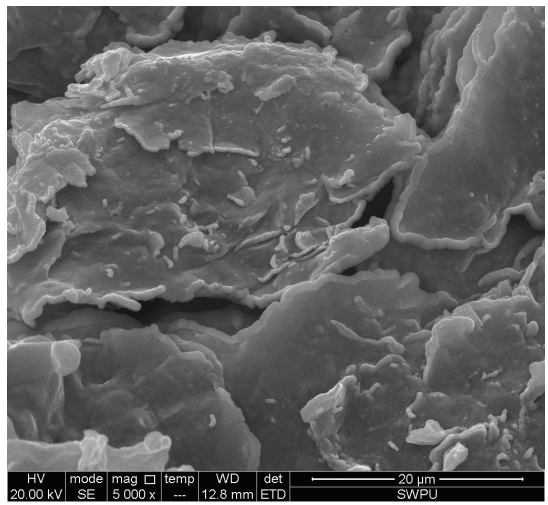

(f)

Figure 5: SEM images of GO (a), GO-OA (b), GO-DDA (c), GO-TDA (d), GO-HAD (e), and GO-ODA (f).

hydrocarbon chain fell off from the basal or edge of GO sheets.

The surface morphologies of GO and M-GO were characterized using SEM. According to SEM images (Figure 5), compared with the GO, the M-GO exhibited an enhanced surface roughness. And the roughness was in the following order: $\mathrm{GO}<\mathrm{GO}-\mathrm{OA}<\mathrm{GO}-\mathrm{DDA}<\mathrm{GO}-\mathrm{TDA}<\mathrm{GO}-\mathrm{HAD}<\mathrm{GO}$ ODA, suggesting a rise in surface roughness with increasing alkyl chain length grafted on GO surface. For the microinterface, the rougher the interface, the more the hydrophobicity. Thus, significant surface roughness could be produced, which was critical for fabricating hydrophobic surface. It offered the possibility of drag reduction in low permeability reservoirs.

Raman spectroscopy was a powerful approach to characterize the graphite degree of carbon-based material. Figure 6 showed the Raman spectra of graphite, GO, and M-GO. After oxidizing, the D band of GO located at $1347 \mathrm{~cm}^{-1}$ increased obviously, which resulted from the decrease in the size of the in-phase $s p^{2}$ domains due to the extensive oxidation. The G band of GO became much broader and shifted to $1596 \mathrm{~cm}^{-1}$ owing to the present of isolated double bonds that resonated at the higher frequencies than the $\mathrm{G}$ band of graphite. The $\mathrm{G}$ band positions of $\mathrm{M}-\mathrm{GO}$ were red shifted relative to pristine $\mathrm{GO}$, owing to the reduction of oxygen-containing groups after grafting long-chain alkyl on the GO surface. The results coincided with the results of XRD and TGA. The
TABLE 1: $I_{\mathrm{D}} / I_{\mathrm{G}}$ of graphite, GO, and M-GO in Raman spectra.

\begin{tabular}{lc}
\hline Samples & $I_{\mathrm{D}} / I_{\mathrm{G}}$ \\
\hline Graphite & 0.1218 \\
GO & 0.9638 \\
GO-OA & 1.0301 \\
GO-DDA & 0.9993 \\
GO-TDA & 0.9897 \\
GO-HDA & 0.9564 \\
GO-ODA & 1.0017 \\
\hline
\end{tabular}

$I_{\mathrm{D}} / I_{\mathrm{G}}$ ratio (where $I_{\mathrm{D}}$ and $I_{\mathrm{G}}$ were the D-peak and G-peak Raman intensity, resp.) was widely used to evaluate the quality of graphene and graphene-based materials. The $I_{\mathrm{D}} / I_{\mathrm{G}}$ ratio values of graphite, GO, and M-GO were shown in Table 1. There was no significant variation in the $I_{\mathrm{D}} / I_{\mathrm{G}}$ ratio for $\mathrm{M}$ $\mathrm{GO}$ when compared to pristine $\mathrm{GO}$, revealing that grafting alkyl on the GO surface did not affect the graphitic structure. The $I_{\mathrm{D}} / I_{\mathrm{G}}$ ratio of $\mathrm{M}-\mathrm{GO}$ increased slightly indicating that the regularity decreased due to the long-chain alkyl increasing the interplanar spacing.

The wetting properties of M-GO were analyzed by measuring the water droplet contact angle on the surface of cores, shown in Figure 7. As for the core sample untreated with $\mathrm{M}$ $\mathrm{GO}$, the water droplet spread rapidly as soon as it contacted 


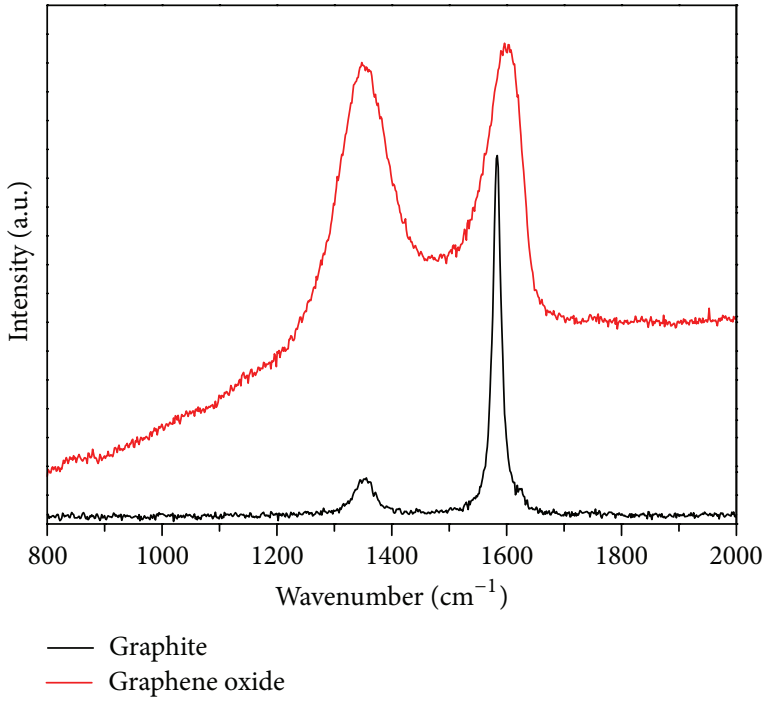

(a)

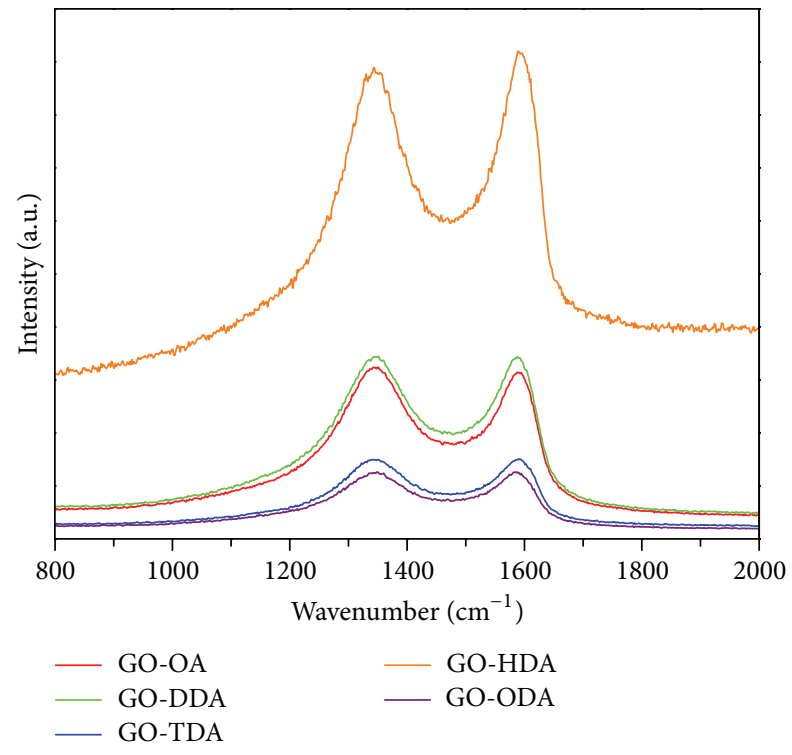

(b)

FIGURE 6: (a) Raman spectra of graphite and graphene oxide. (b) Raman spectra of M-GO.

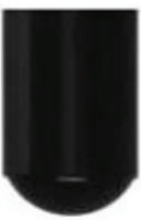

CA: $107.53^{\circ}$

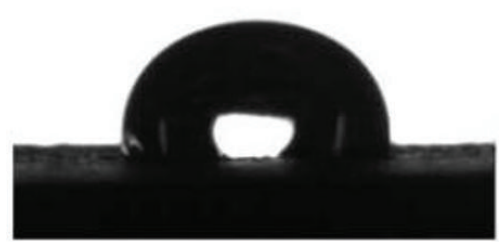

GO-OA

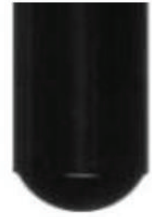

CA: $111.92^{\circ}$

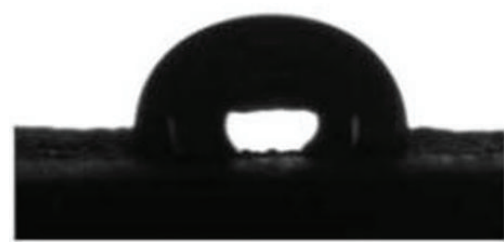

GO-DDA

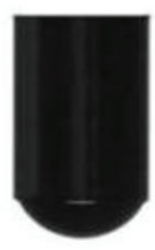

CA: $114.35^{\circ}$

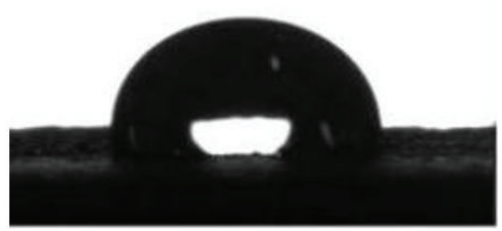

GO-TDA

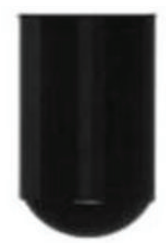

CA: $116.09^{\circ}$

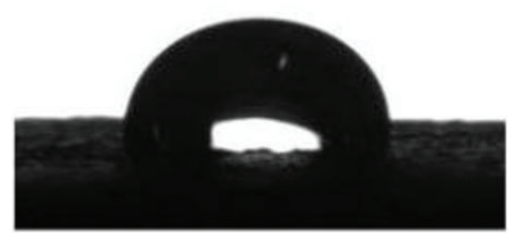

GO-HDA

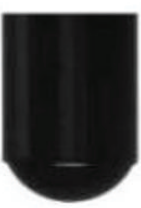

CA: $118.97^{\circ}$

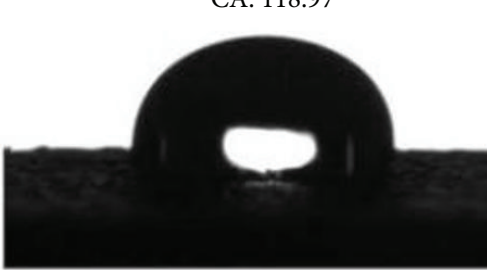

GO-ODA

Figure 7: Contact angle on surface of the cores. 
TABle 2: Parameter of cores and experiment.

\begin{tabular}{lccccccc}
\hline Number & $\begin{array}{c}\text { Size } \\
(\mathrm{cm})\end{array}$ & $\begin{array}{c}\text { Pore volume } \\
(\mathrm{mL})\end{array}$ & $\begin{array}{c}\text { Porosity } \\
(\%)\end{array}$ & $\begin{array}{c}\text { Permeability } \\
\left(\times 10^{-3} \mu \mathrm{m}^{2}\right)\end{array}$ & $\begin{array}{c}\text { Injection rate } \\
\mathrm{mL} / \mathrm{min}\end{array}$ & $\begin{array}{c}\text { Concentration } \\
(\mathrm{mg} / \mathrm{L})\end{array}$ & $\begin{array}{c}\text { Injection } \\
\text { volume } \\
(\mathrm{PV})\end{array}$ \\
\hline 1 & $\Phi 2.5 \times 8$ & 7.8 & 19.9 & 22.5 & & & $\begin{array}{c}\text { Time } \\
(\mathrm{h})\end{array}$ \\
2 & $\Phi 2.5 \times 8$ & 8.0 & 20.4 & 27.3 & 1 & 50 & 1 \\
3 & $\Phi 2.5 \times 8$ & 7.9 & 20.1 & 28.1 & & 48 \\
4 & $\Phi 2.5 \times 8$ & 7.8 & 19.9 & 23.2 & & \\
\hline
\end{tabular}

TABLE 3: Pressure drop and permeability ratio before and after nanodrag reducer injection.

\begin{tabular}{|c|c|c|c|c|c|c|}
\hline Materials & $\begin{array}{c}P_{1} \\
(\mathrm{MPa})\end{array}$ & $\begin{array}{c}P_{2} \\
(\mathrm{MPa})\end{array}$ & $\begin{array}{c}K_{1} \\
\left(\times 10^{-3} \mu \mathrm{m}^{2}\right)\end{array}$ & $\begin{array}{c}K_{2} \\
\left(\times 10^{-3} \mu \mathrm{m}^{2}\right)\end{array}$ & $\begin{array}{c}\text { Pressure drop rate } \\
(\%)\end{array}$ & $K_{2} / K_{1}$ \\
\hline GO-DDA & 0.2864 & 0.3682 & 5.70 & 4.43 & -28.56 & 0.78 \\
\hline GO-TDA & 0.2879 & 0.3017 & 5.66 & 5.40 & -4.79 & 0.95 \\
\hline GO-HDA & 0.2925 & 0.2477 & 5.57 & 6.58 & 15.32 & 1.18 \\
\hline GO-ODA & 0.2876 & 0.1566 & 5.56 & 10.41 & 45.55 & 1.84 \\
\hline
\end{tabular}

the surface of core due to the capillarity phenomenon; the $\mathrm{CA}$ of core could be considered as $0^{\circ}$. So the cores were hydrophilic. After M-GO deposited on the surface of core, the contact angles of cores were $107.53^{\circ}, 111.92^{\circ}, 114.35^{\circ}$, $116.09^{\circ}$, and $118.97^{\circ}$, respectively. The grafting of alkylamines increased the water contact angle that may be due to the increase of hydrophobicity as well as surface roughness of the GO.

The M-GO would produce two important effects after being injected into the cores. In the first one, it would adsorb on the pore throats' surface of the rock, the wettability of the surface changed from hydrophilicity to hydrophobicity, then the slip effect appeared, and the flow resistance reduced. In the other one, the M-GO would reduce the diameter of pore throats after adsorbing on the surface, causing the water injection pressure to increase. Therefore, whether $\mathrm{M}-\mathrm{GO}$ could reduce the water injection pressure or not depended on both the overall effects. If the nano slip effect was dominant, the flow resistance and injection pressure would decrease and vice versa.

The core flooding experiments were carried out to evaluate the ability of drag reduction of $\mathrm{M}-\mathrm{GO}$ for low permeability. The GO-OA was unable to disperse in diesel oil completely to form homogeneous dispersion, so we only analyzed the drag reduction effect of GO-DDA, GOTDA, GO-HAD, and GO-ODA. The cores and experiment parameters were listed in Table 2 and the pressure curve of flooding was shown in Figure 8. According to the pressure of the four kinds of nanodrag reducer, the permeability was calculated with Darcy equation (Table 3). With the increase of alkyl chain length on the GO surface, the decompression and augmented injection effect of nanodrag reducer was better. After M-GO injected into cores, the subsequent water flooding pressure of GO-DDA and GO-TDA became higher than before, which could not lower water injection pressure, while the subsequent water flooding pressure of GO-HAD and GO-ODA decreased $15.32 \%$ and $45.55 \%$, respectively, and

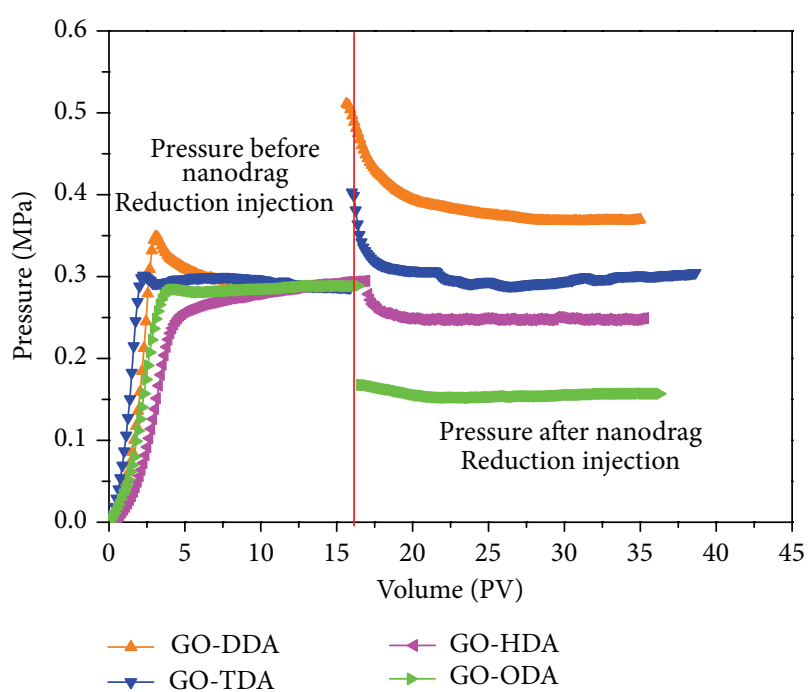

FIGURE 8: Pressure of water flooding before and after nanodrag reducer injection.

the permeability was 1.18 and 1.84 times higher than before, so GO-DDA and GO-TDA could be used as water flooding nanodrag reducer agent for low permeability reservoirs.

\section{Conclusions}

In summary, modified graphene oxide (M-GO) was synthesized by grafting long alkylamines chain with varying lengths, which could be used as nanodrag reducer in oilfield water flooding. We utilized FTIR, XRD, TGA, SEM, and Raman spectra measurements to characterize the successful modification of M-GO. SEM revealed that the long hydrocarbon chain in alkylamines increased the GO surface roughness. Water contact angle measurements results indicated that 
the chain length of the grafted alkylamine was crucial for increasing the hydrophobicity of the GO, which maybe corroborated the decrease in surface energy values and increase in surface roughness. Flooding experiments showed that the GO-HDA and GO-ODA had an ability to reduce water injection pressure and increase water injection volume in the low permeability reservoirs. Our work may lay foundation for further industrial applications of modified GO in oilfield exploitation.

\section{Disclosure}

The experiments were performed at the State Key Laboratory of Oil and Gas Reservoir Geology and Exploitation of Southwest Petroleum University in Chengdu in China.

\section{Competing Interests}

The authors declare that they have no competing interests regarding the publication of this paper.

\section{Acknowledgments}

This work was supported by the Petroleum Technology Innovation Found of CNPC (no. 2011D-5006-0209).

\section{References}

[1] J. I. Parades, S. Villar-Rodil, A. Martínez-Alonso, and J. M. D. Tascón, "Graphene oxide dispersions in organic solvents," Langmuir, vol. 24, no. 19, pp. 10560-10564, 2008.

[2] K. I. Bolotin, K. J. Sikes, Z. Jiang et al., "Ultrahigh electron mobility in suspended graphene," Solid State Communications, vol. 146, no. 9-10, pp. 351-355, 2008.

[3] K. S. Novoselov, D. Jiang, F. Schedin et al., "Two-dimensional atomic crystals," Proceedings of the National Academy of Sciences of the United States of America, vol. 102, no. 30, pp. 10451-10453, 2005.

[4] A. K. Geim and K. S. Novoselov, "The rise of graphene," Nature Materials, vol. 6, no. 3, pp. 183-191, 2007.

[5] A. A. Balandin, S. Ghosh, W. Z. Bao et al., "Superior thermal conductivity of single-layer graphene," Nano Letters, vol. 8, no. 3, pp. 902-907, 2008.

[6] Y. B. Zhang, Y.-W. Tan, H. L. Stormer, and P. Kim, "Experimental observation of the quantum Hall effect and Berry's phase in graphene," Nature, vol. 438, no. 7065, pp. 201-204, 2005.

[7] Z. Qian, Z. C. Zhang, L. Y. Song, and H. R. Liu, "A novel approach to raspberry-like particles for superhydrophobic materials," Journal of Materials Chemistry, vol. 19, no. 9, pp. 1297-1304, 2009.

[8] C. Lee, X. Wei, J. W. Kysar, and J. Hone, "Measurement of the elastic properties and intrinsic strength of monolayer graphene," Science, vol. 321, no. 5887, pp. 385-388, 2008.

[9] H. Chen, M. B. Müller, K. J. Gilmore, G. G. Wallace, and D. Li, "Mechanically strong, electrically conductive, and biocompatible graphene paper," Advanced Materials, vol. 20, no. 18, pp. 3557-3561, 2008.

[10] T. Huang, R. Lu, C. Su et al., "Chemically modified graphene/ polyimide composite films based on utilization of covalent bonding and oriented distribution," ACS Applied Materials and Interfaces, vol. 4, no. 5, pp. 2699-2708, 2012.

[11] N. D. Luong, N. Pahimanolis, U. Hippi et al., "Graphene/ cellulose nanocomposite paper with high electrical and mechanical performances," Journal of Materials Chemistry, vol. 21, no. 36, pp. 13991-13998, 2011.

[12] H. Tang, G. J. Lin, and H. A. Sodano, "Highly efficient synthesis of graphene nanocomposites," Nano Letters, vol. 12, no. 1, pp. 84-90, 2011.

[13] J. Y. Kim, L. J. Cote, and J. X. Huang, "Two dimensional soft material: new faces of graphene oxide," Accounts of Chemical Research, vol. 45, no. 8, pp. 1356-1364, 2012.

[14] S. Stankovich, D. A. Dikin, R. D. Piner et al., "Synthesis of graphene-based nanosheets via chemical reduction of exfoliated graphite oxide," Carbon, vol. 45, no. 7, pp. 1558-1565, 2007.

[15] N. Li, S. Tang, Y. Dai, and X. Meng, "The synthesis of graphene oxide nanostructures for supercapacitors: a simple route," Journal of Materials Science, vol. 49, no. 7, pp. 2802-2809, 2014.

[16] W.-S. Ma, J. Li, B.-J. Deng, and X.-S. Zhao, "Preparation and characterization of long-chain alkyl silane-functionalized graphene film," Journal of Materials Science, vol. 48, no. 1, pp. 156-161, 2013.

[17] P. G. Liu, K. C. Gong, P. Xiao, and M. Xiao, "Preparation and characterization of poly(vinyl acetate)-intercalated graphite oxide nanocomposite," Journal of Materials Chemistry, vol. 10, no. 4, pp. 933-935, 2000.

[18] A. Lerf, H. He, M. Forster, and J. Klinowski, "Structure of graphite oxide revisited," Journal of Physical Chemistry B, vol. 102, no. 23, pp. 4477-4482, 1998.

[19] S. Park, K.-S. Lee, G. Bozoklu, W. Cai, S. T. Nguyen, and R. S. Ruoff, "Graphene oxide papers modified by divalent ionsenhancing mechanical properties via chemical cross-linking," ACS Nano, vol. 2, no. 3, pp. 572-578, 2008.

[20] H. Wang, P.-G. Ren, J.-Z. Xu, D.-X. Yan, Z.-M. Li, and L. Xu, "Non-isothermal crystallization kinetics of alkyl-functionalized graphene oxide/high-density polyethylene nanocomposites," Composite Interfaces, vol. 21, no. 3, pp. 203-215, 2014.

[21] T. Kuila, S. Bose, C. E. Hong et al., "Preparation of functionalized graphene/linear low density polyethylene composites by a solution mixing method," Carbon, vol. 49, no. 3, pp. 1033-1037, 2011.

[22] X. J. Feng and L. Jiang, "Design and creation of superwetting/antiwetting surfaces," Advanced Materials, vol. 18, no. 23, pp. 3063-3078, 2006.

[23] A. M. Shanmugharaj, J. H. Yoon, W. J. Yang, and S. H. Ryu, "Synthesis, characterization, and surface wettability properties of amine functionalized graphene oxide films with varying amine chain lengths," Journal of Colloid and Interface Science, vol. 401, pp. 148-154, 2013.

[24] J. Liu, Y. Wang, S. Xu, and D. D. Sun, "Synthesis of graphene soluble in organic solvents by simultaneous etherfunctionalization with octadecane groups and reduction," Materials Letters, vol. 64, no. 20, pp. 2236-2239, 2010.

[25] J. Rafiee, M. A. Rafiee, Z.-Z. Yu, and N. Koratkar, "Superhydrophobic to superhydrophilic wetting control in graphene films," Advanced Materials, vol. 22, no. 19, pp. 2151-2154, 2010.

[26] X. Q. Zhang, S. H. Wan, J. B. Pu, L. P. Wang, and X. Q. Liu, "Highly hydrophobic and adhesive performance of graphene films," Journal of Materials Chemistry, vol. 21, no. 33, pp. 1225112258, 2011. 
[27] Z. Y. Lin, Y. Liu, and C.-P. Wong, "Facile fabrication of superhydrophobic octadecylamine-functionalized graphite oxide film," Langmuir, vol. 26, no. 20, pp. 16110-16114, 2010.

[28] Z.-B. Ye, Y. Xu, H. Chen, C. Cheng, L.-J. Han, and L. Xiao, "A novel micro-nano structure profile control agent: graphene oxide dispersion," Journal of Nanomaterials, vol. 2014, Article ID 582089, 9 pages, 2014. 

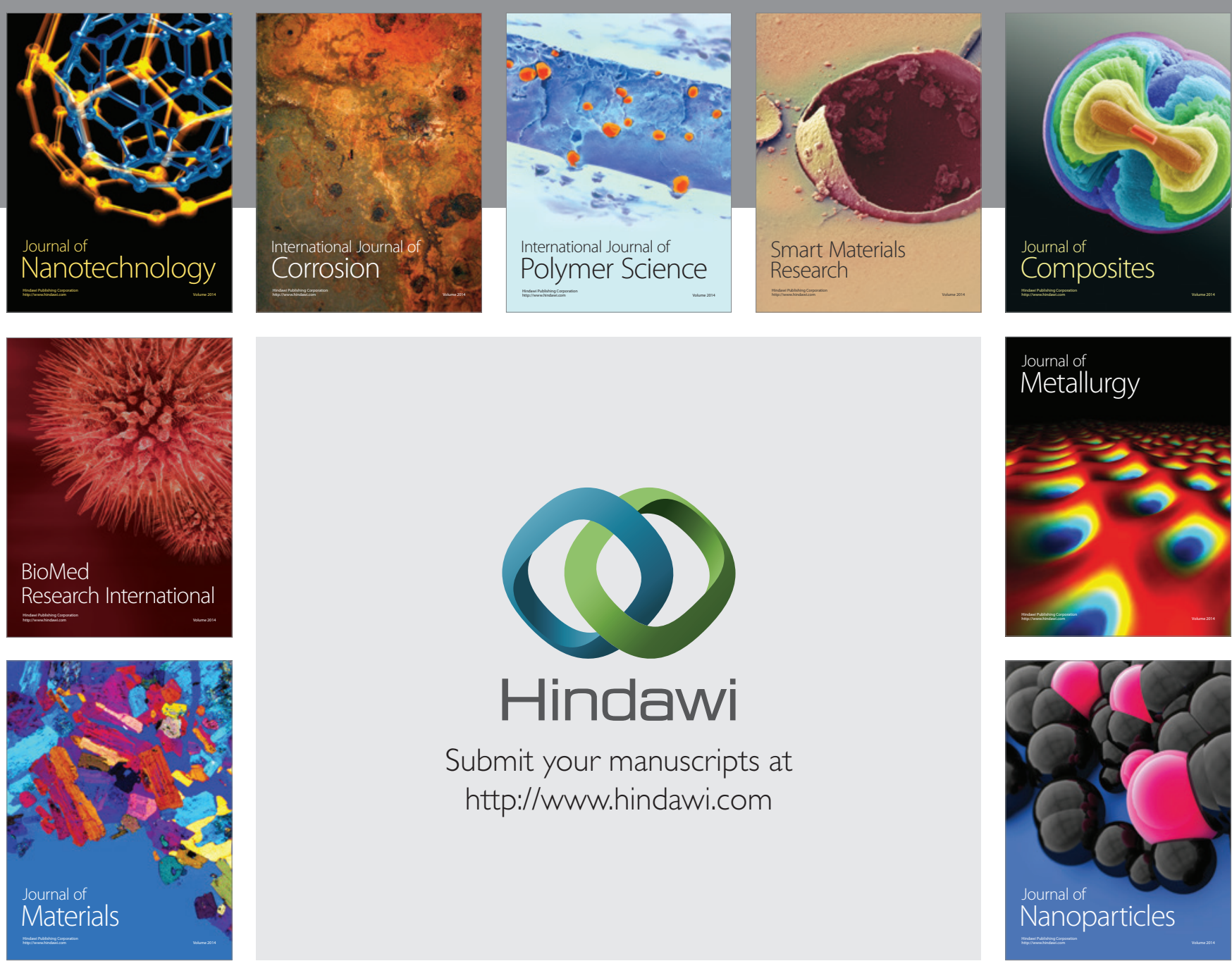

\section{Hindawi}

Submit your manuscripts at

http://www.hindawi.com

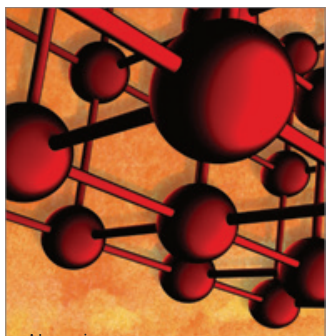

Materials Science and Engineering
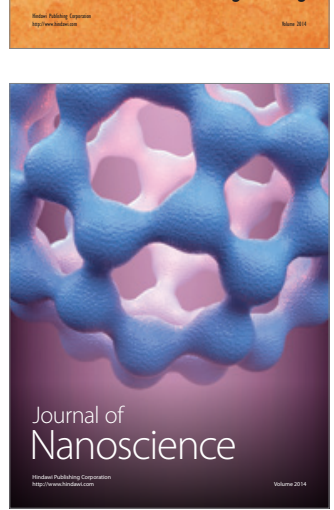
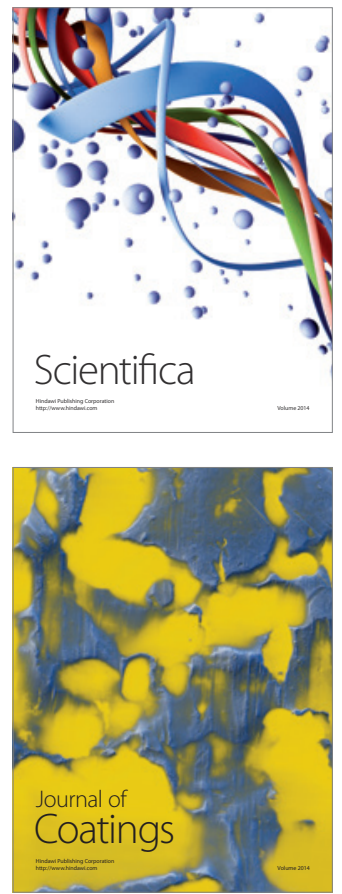
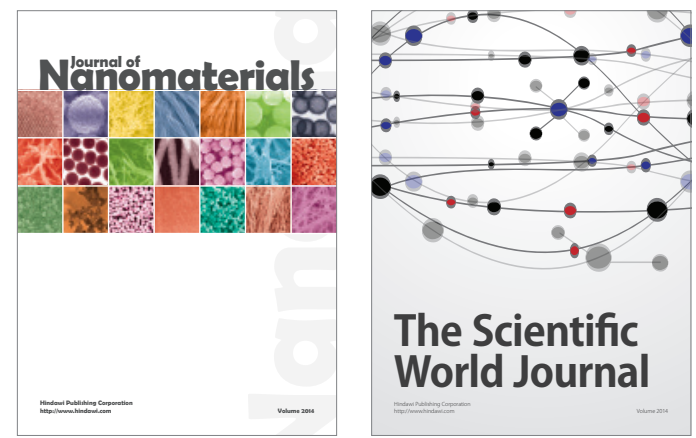

The Scientific World Journal
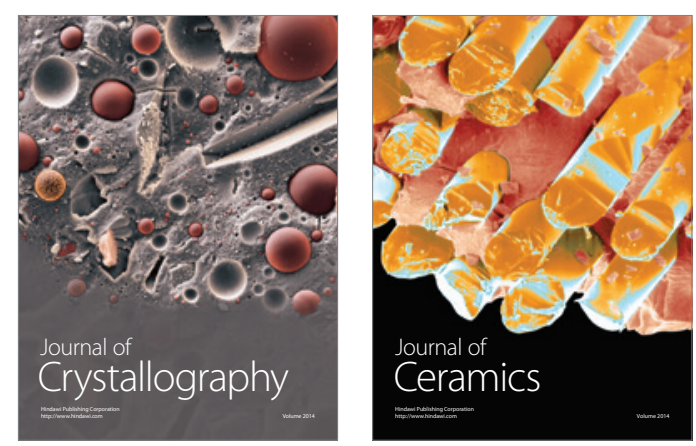
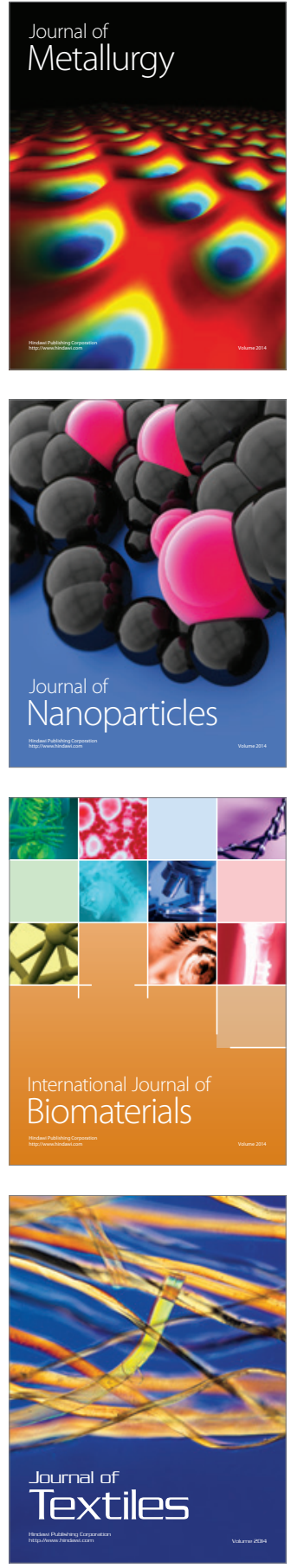